\title{
Design of Deer Breeding Management System Based on Internet of Things
}

\author{
Min Zhang a, Yuhua Wu, Ji Li, Shijun Li ${ }^{b,}$ * \\ Jilin Agricultural University, Changchun 130118, Jilin, China \\ a1165339875@qq.com, b Isj0883@sina.com \\ Corresponding Author: Shijun Li
}

Keywords: Deer breeding, RFID, Internet of Things, ZigBee.

\begin{abstract}
This paper use the internet of things technology, RFID technology, wireless communication technology to design and achieve the standardized management system for improving standardized breeding level of deer. The system collects and records the information of deer growth status, population, diseases, antlers. At the same time, it transfers these data that generated in each link to system server by wireless communication network, Internet network. Then the data are analyzed and organized by system. It is convenient for the farmers to track, query the growth of deer. Thus, the system can improve the standardized level of deer breeding, improving the yield and quality of deer related products.
\end{abstract}

\section{Introduction}

China has a long history of deer farming, first appeared in the Shang and Zhou Dynasties, but also one of the earliest countries in the world[1]. Especially after the founding of the people's Republic of China, it has realized the large-scale breeding of deer, which has been exported to the international market for a long period of time[2]. Because most of the deer farm farming still rely on manual handling, standardization, and scientific level is not high, resulting in high cost. However New Zealand use of standardized, scientific farming methods, the antler export occupy the international market share of $70 \%[3,4]$.

With the development of Internet of things technology, RFID technology and related technology, Internet of Things technology plays an important role in the standardization and scientific development of aquaculture industry. At present, the Internet of things technology has achieved great success in the breeding industry. Realization of beef quality safety traceability system by using Internet of Things technology and RFID technology[5-7]. Through the use of RFID and wireless sensor network technology combined intelligent monitoring of aquaculture aquaculture[8]. Through RFID and ZigBee Technology to Realize Information Management of Sheep Culture[9,10].

Therefore, this paper uses the Internet of things technology, RFID technology, wireless communication technology design and implementation of the deer standardized breeding system. Through the wireless communication network, the Internet and other transmission methods, the deer growth-related information uploaded to the server, farmers can be timely through the client to grasp the growth of deer, so as to improve the standardization of deer breeding, improve deer breeding quality and production.

\section{System Design}

\subsection{The Basic Structure of the System}

The overall framework of the system is shown in Figure 1, the standardized management system can be divided into information collection layer, information transmission layer and application management platform. Through the use of RFID tags and reader equipment can write and read the deer field name, weight, disease prevention and control situation, deer only change the situation, deer and other related information through the information collection node to send information to the 
coordinator side. Then connected to the Internet, the deer growth information sent to the application management platform, the farmer can view and manage the deer's growth information through the client software.

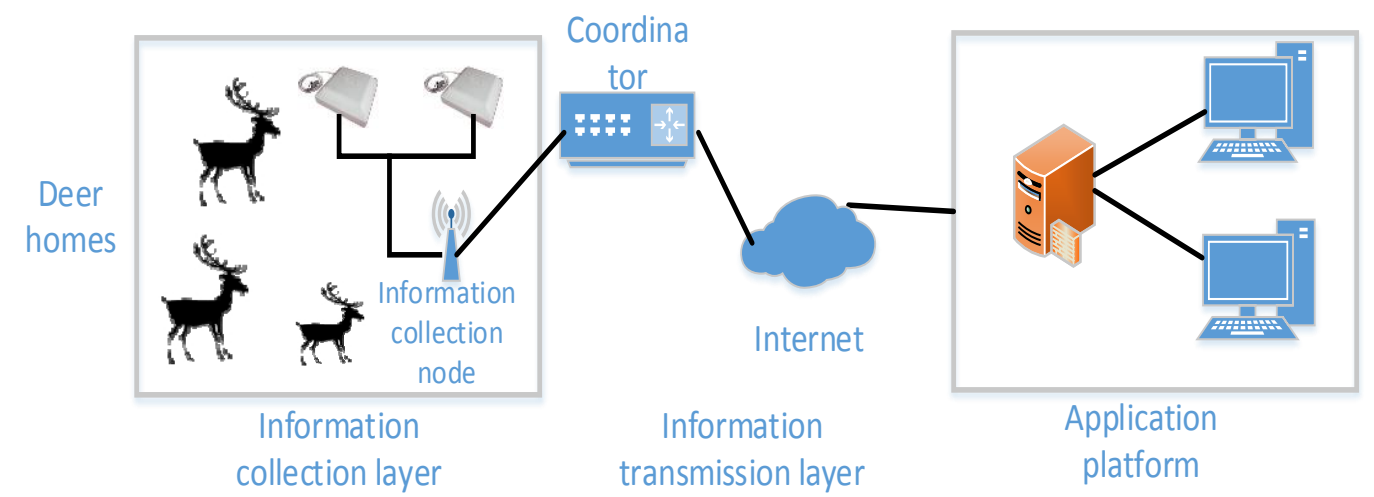

\subsection{Deer Logo System}

Fig. 1 The overall framework of the system

The use of radio frequency identification technology (RFID) to distinguish between different objects and the corresponding data collection. At present, the development of RFID technology is high, one time not only can identify a single object, but also when there are multiple objects can also be accurately identified. Because of its movement of objects can be identified, and in the process of collecting information without the need for contact information can be collected [11], so the application of RFID in the livestock industry is also very extensive. The RFID system block diagram shown in Figure 2.

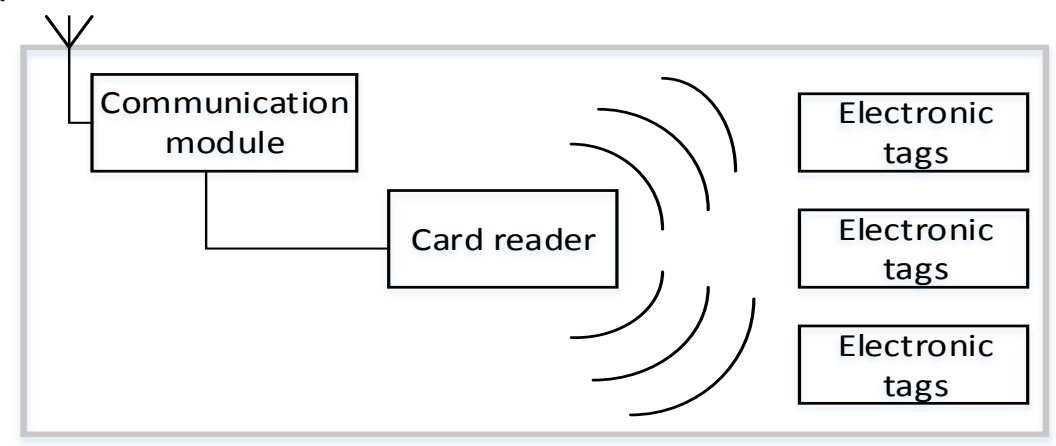

Fig. 2 RFID system block diagram

The RFID system consists of electronic tag and reader, the electronic tag has a shockproof, durable, anti-electromagnetic wave etc, the installation is very convenient, and the stored data can not be lost easily, can be used for intelligent tracking management of each deer. Each deer using their own portable RFID earcon record basic information, including varieties, origin, distribution of deer farm information. The RFID ear tag according to the daily feeding situation of deer, disease prevention and treatment time and the results of establishing electronic archives, and timely modify the relevant information recorded in the ear tag, synchronization of information recorded in the management system, the growth of convenient aquaculture personnel tracking management of deer.

\subsection{Wireless Communication Technology}

The system uses ZigBee technology for wireless communication. ZigBee is a kind of wireless communication technology which can carry out short distance information transmission and low energy consumption [12]. It is a communication platform which is composed of multiple wireless data transmission modules. ZigBee communication can be divided into two main parts of router and coordinator, the coordinator is mainly responsible for the establishment of the network and the network initialization settings, but only in a ZigBee network allows for a coordinated, and at the same time networking and communication. The router is responsible for the information transmission between the reader and coordinator of the RFID, and then access to the Internet through the coordinator, and the information is transferred to the application platform through the coordinator.

The ZigBee-based communication scheme shown in Figure 3. 


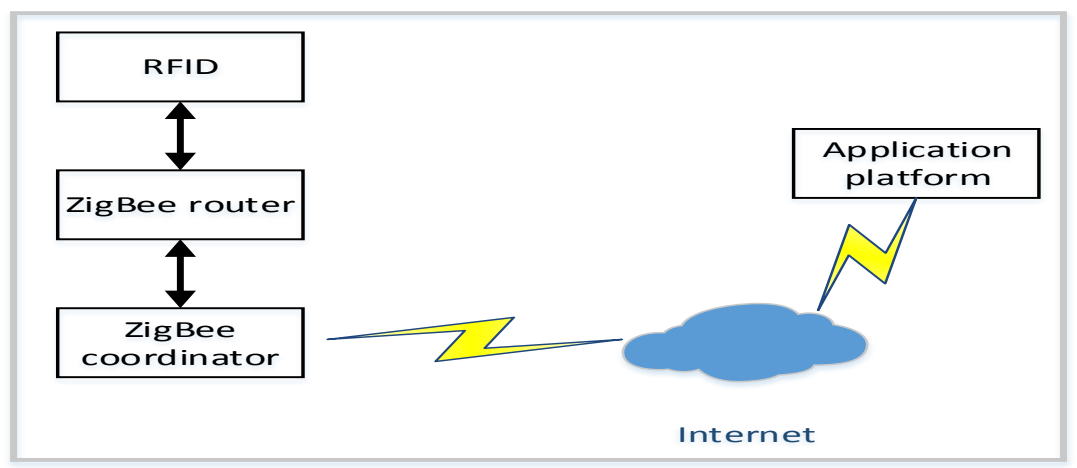

Fig. 3 ZigBee-based communication scheme

\section{Design of ZigBee Communication Software}

In this system, the ZigBee communication module uses JN5168 for data transmission. The JN5168 network is used to start the coordinator node first, power the nodes in the network and initialize the whole network, and then start the whole network by running the Jen OS operating system carried by JN5168. It is able to receive the network request signal of the sub-network, when the whole network is established, the coordinator will be converted into a routing with the transceiver function [13]. And then start the routing node of the sub-network, when the routing node will send the network request signal to the entire network, query the coordinator in the network and request the network, the coordinator receives and allows the routing node to issue the network request, the routing node will be added to this communication network. The JN5168 communication flow chart shown in Figure 4.

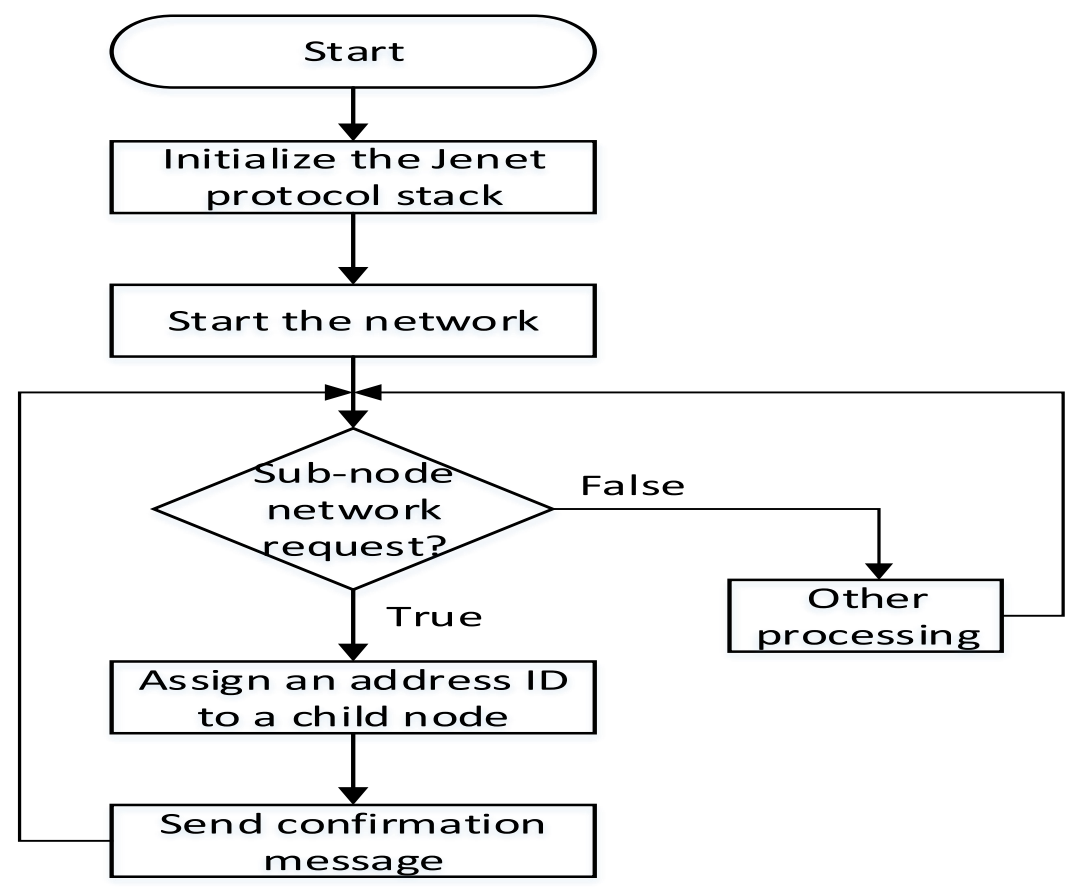

Fig. 4 JN5168 communication flow chart

\section{System Implementation}

The whole system runs under Windows, the farmer through the user name and password to log in the system, the first sub-field registration, enter the corresponding sub-field name and deer varieties, before the deer can be related information entry operation. The deer's growth status and change information are recorded by deer registration, disease management, deer change and so on, and the information file is established by statistical analysis. Such as deer only registered modules include deer only information registration, deer information query, deer change information query function. Record the deer's growth process information, and then establish an electronic file, you can facilitate the 
breeding staff to check deer in the deer's growth information and deer only change the information, and can generate print forms. Such as the deer registration need to select the appropriate deer field information, and then record the basic characteristics of deer, velvet records, body weight, graphic photos. Deer basic characteristics of information, including deer field name, field, deer, deer varieties, birth time, origin and other related information.

\section{Conclusion}

Combining traditional deer farming and networking technology, RFID technology and wireless communication technology, the design of RFID-based deer standardized culture management system is designed. The system can record the deer's growth-related information in real time and establish electronic files of related information such as population, disease prevention, breeding, production and so on. It can facilitate the breeding of trainees, check the growth of deer, improving the standardization level of traditional deer breeding Aquaculture production and quality.

\section{Acknowledgments}

The authors wish to express their gratitude to the projects: Design of Deer Breeding Management System Based on Internet of Things from Education Department of Jilin Province, Jilin Province Economic Structural Adjustment Leading Fund Special Project (No. 2014Y108) and Changchun City Science and Technology Plan Project (No. 14nk029), Jilin Province Science and Technology Development Program Funded Project (No. 20160623016TC), Education Department of Jilin Province's 12th Five-Year Science and Technology Research Project (2015175), for their generous support of this work. At the same time, the other authors also thank the corresponding author, Shijun LI, for his assistance in submitting this paper.

\section{References}

[1]. JIANG Yifei, LI Feng, ZHANG Shidong, et al. Present situation and Prospect of sika deer industry in China [J]. Shandong Agricultural Sciences, 2012, (09):109-111+114.

[2]. LIU Yan, ZHENG Ce, ZHANG Xu, et al. Study on Present Situation and Development Countermeasures of Velvet Deer Farming in China [J]. Chinese Journal of Animal Science, 2011, (12):18-21.

[3]. ZHANG Zhenxing. Current situation and development strategy of deer industry in China [J]. Journal of Economic Animal, 2003, (02):1-4.

[4]. TANG Rui. Study on the present situation and strategy of deer industry in China [D]. Jilin University, 2013.

[5]. LIU Peng, LIU Wen, Li Qiang, et al. Establishment of Cattle Breeding System Based on RFID Technology[J].Food Research and Development,2015,(24):190-193.

[6]. CHENG Jing, JIA Yinjiang, GUAN Jing. Application of RFID Middleware Incattle Breeding Traceability System[J]. Journal of Agricultural Mechanization Research, 2015, (05):224-228.

[7]. SHI Liang, FU Zetian, ZHANG Lingxian. Research on Quality and Traceability System of Beef Cattle Breeding Based on RFID Technology [J]. Computer Applications and Software, 2010, (01):40-43.

[8]. YAN Bo, SHI Ping. Intelligent Monitoring System for Aquiculture Based on Internet of Things [J]. Transactions of the Chinese Society for Agricultural Machinery, 2014, (01):259-265.

[9]. WANG Ling, ZOU Xiaoyi, LIU Siyao, et al. Development of Handheld Terminal for Sheep Breeding Information Management Based on RFID and ZigBee [J]. Transactions of the Chinese Society for Agricultural Machinery, 2014, (9):247-253.

[10]. ZHENG Kui. Design and Implementation of Embedded Handheld Terminal Based on RFID and ZigBee's Sheep Culture Information Management [D]. Nanjing Agricultural University, 2013. 
[11]. WANG Yawei. A Summary of the Development of Internet of Things [J]. Science \& Technology Information, 2010, (3):54-56.

[12]. LIU Na, MA Li. A Novel WSN Data Acquiring System based on ZigBee [J]. Computer Development \& Applications, 2010, (06):69-70.

[13]. Bu Yanru, Cheng Hai, Zhai Shuxia. Design of greenhouse monitoring and controlling system based on JN5168 [J]. Journal of Chinese Agricultural Mechanization, 2014, (04):208-211. 